\title{
Role of nanotechnology and gene delivery systems in TRAIL-based therapies
}

\author{
George E Naoum 1 , Fady Tawadros ${ }^{2}$, Ammad Ahmad Farooqi $^{3}$, Muhammad Zahid Qureshi ${ }^{3}$, Sobia Tabassum $^{3}$, \\ Donald J Buchsbaum ${ }^{4}$ and Waleed Arafat ${ }^{4,5}$
}

\author{
${ }^{1}$ Alexandria Comprehensive Cancer Centre, Egypt \\ ${ }^{2}$ East Tennessee State University, 1276 Gilbreath Dr, Johnson City, TN 37604, USA \\ ${ }^{3}$ Institute of Biomedical and Genetic Engineering (IBGE), Islamabad, Pakistan \\ ${ }^{4}$ University of Alabama at Birmingham, 1720 2nd Ave S, Birmingham, AL 35233, USA \\ ${ }^{5}$ University of Alexandria, El-Gaish Rd, Egypt, Alexandria, Egypt
}

Correspondence to: Waleed Arafat. Email: w.o.arafaat@gmail.com

\begin{abstract}
Since its identification as a member of the tumour necrosis factor (TNF) family, TRAIL (TNF-related apoptosis-inducing ligand) has emerged as a new avenue in apoptosis-inducing cancer therapies. Its ability to circumvent the chemoresistance of conventional therapeutics and to interact with cancer stem cells (CSCs) self-renewal pathways, amplified its potential as a cancer apoptotic agent. Many recombinant preparations of this death ligand and monoclonal antibodies targeting its death receptors have been tested in monotherapy and combinational clinical trials. Gene therapy is a new approach for cancer treatment which implies viral or non-viral functional transgene induction of apoptosis in cancer cells or repair of the underlying genetic abnormality on a molecular level. The role of this approach in overcoming the traditional barriers of radiation and chemotherapeutics systemic toxicity, risk of recurrence, and metastasis made it a promising platform for cancer treatment. The recent first Food Drug Administration (FDA) approved oncolytic herpes virus for melanoma treatment brings forth the potency of the cancer gene therapy approach in the future. Many gene delivery systems have been studied for intratumoural TRAIL gene delivery alone or in combination with chemotherapeutic agents to produce synergistic cancer cytotoxicity. However, there still remain many obstacles to be conquered for this different gene delivery systems. Nanomedicine on the other hand offers a new frontier for clinical trials and biomedical research. The FDA approved nanodrugs motivates horizon exploration for other nanoscale designed particles' implications in gene delivery. In this review we aim to highlight the molecular role of TRAIL in apoptosis and interaction with cancer stem cells (CSCs) self-renewal pathways. Finally, we also aim to discuss the different roles of gene delivery systems, mesenchymal cells, and nanotechnology designs in TRAIL gene delivery.
\end{abstract}

Keywords: TRAIL, nanotechnology, cancer gene therapy, apoptosis

Published: 01/08/2016

Received: $31 / 03 / 2016$

ecancer 2016, 10:660 DOI: 10.3332/ecancer.2016.660

Copyright: $\odot$ the authors; licensee ecancermedicalscience. This is an Open Access article distributed under the terms of the Creative Commons Attribution License (http://creativecommons.org/licenses/by/3.0), which permits unrestricted use, distribution, and reproduction in any medium, provided the original work is properly cited. 


\section{Introduction}

The symphony of balance between cell growth and cell death,in order to preserve normal biological functional environment, is maintained through orchestrated processes. Among these processes is apoptosis which is programmed cell death [1]. Countless researchers have highlighted that any interference with this balance can lead to cancer development [2]. The process of apoptosis itself is still an extremely complicated process in which many molecules and cellular pathways are involved [3]. In this review we aim to focus on TRAIL, a leading molecule involved in the process of apoptosis and its promising effects as apoptosis inducer for cancer treatment. We also aim to highlight the role of different gene delivery systems as alternative approaches to conventional TRAIL-based therapies in cancer.

\section{TRAIL a holy grail to apoptosis}

Since the discovery of TNF family members, a new milestone in apoptosis has emerged. During the 1990s, Wiley et al and Pitti et al identified a new member of the TNF named TRAIL (tumour necrosis factor-related apoptosis-inducing ligand) or Apo 2 ligand [4, 5]. The major biological role of this 281-amino acid type II trans-membrane protein is apoptosis induction, after interacting with its receptors [6-8]. Four different homologous human TRAIL receptors have been classified, these are TRAIL-R1/DR4, TRAIL-R2/ DR5 also known as killer, TRAILR3 or DcR1, and TRAIL-R4 or DcR2 [9-12]. The death receptors DR4 and DR5, both contain a preserved death domain (DD) motif and play an important role in signal apoptosis signalling [13, 14]. The other receptors TRAIL-R3/DcR1 and TRAIL-R4/DcR2 have homology to the DR4 and DR5 extracellular domains and are believed to act as decoys binding to TRAIL and inhibit its effect on apoptosis induction [15].

After binding to its receptor, TRAIL activates two main signalling pathways; the intrinsic and extrinsic pathways for cell death [16].

\section{The extrinsic pathway}

The cell apoptotic extrinsic pathway is initiated when TRAIL binds three receptor molecules of either DR5 or DR4 forming homotrimers, each at the interface between two of its subunits [17-18], like most other TNF family members. This ligation and receptor trimerisation results in aggregation of the receptor's intracellular DD, leading to the formation of a macromolecular complex known as death-inducing signalling complex (DISC); where an adaptor molecule Fas associated death domain protein (FADD) is recruited and binds to caspase-8 and -10 to activates them. The activated caspase- 8 and -10 allow the conversion of pro-caspase- 3 to its active form which in turn leads to cleavage of the death substrates. It is to be noted that this pathway can be inhibited by the cellular FLICE inhibitory protein (c-FLIP). This protein shares homologous sequence with caspase 8 , and thus might compete with it for FADD binding in the DISC, as illustrated in Figure 1B. The presence of the c-FLIP protein in the DISC attenuates its function as it lacks protease activity. In addition to that, in the presence of c-FLIP, DISC forms a secondary complex with receptor-interacting protein (RIP), NFKB kinase, TNFR1-associated death domain (TRADD), and TNF receptor-associated factor 2 (TRAF2). This secondary complex activates non-apoptotic signals initiated through the mitogen-activated protein kinase (MAPK) pathways, nuclear factor $\mathrm{KB}$, and phosphoinositide 3-kinase (PI3K)/Akt.

On the other hand, the two apoptosis signalling pathways,the intrinsic and the extrinsic, communicate with each other [19] as depicted in Figure $1 \mathrm{~A}$.

\section{The intrinsic pathway}

Upon death stimulus, Bid (a member the proapoptotic Bcl-2 family) is cleaved by caspase-8. This cleavage leads to the formation of truncated form of Bid that interact with Bax and Bak in mitochondria to destabilise its outer membrane, so cytochrome $c$ can be released. In the cytosol, an apoptosome is formed by the binding of cytochrome $c$ to the adaptor APAF-1 and pro-caspase-9 as depicted in Figure 1 A. In turn, caspase- 9 after being activated by the apoptosome activates 'executioner' protease caspase $-3,-6$, and -7 . This connection apparently plays a role in amplifying the response to death receptor activation and different types of cells might rely more on this amplification pathway than others [20]. This pathway was found to be inhibited by members of IAP (inhibitory of apoptosis) family including cellular IAP 1 and 2 , X-linked IAP, and survivin. These molecules contribute to TRAIL resistance by inhibiting the activity of caspases 3 , 7, and/or 9 . Nevertheless, Smac 


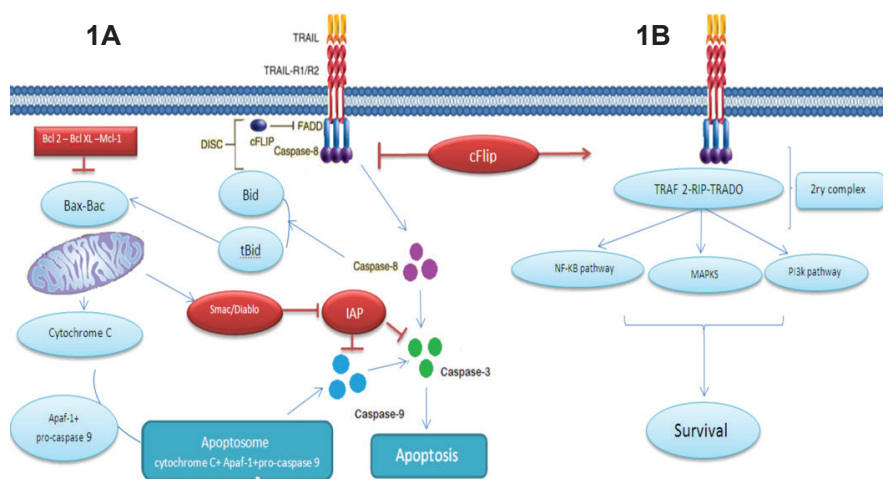

Figure 1. Showing the TRAIL signalling pathway. On the right Figure(1A), the apoptotic pathway induced by TRAIL and to the left Figure (1B), the resistance to TRAIL induced apoptosis. Binding of TRAIL and trimerisation of TRAIL death receptors leads to recruitment of FADD, an adaptor molecule that is capable of caspase-8 recruitment and activation. Apoptosis is either induced through direct caspase-8-mediated caspase-3 activation or through an amplification loop involving the mitochondria and the cleavage of the BH3-only protein Bid by caspase-8, cFLIP interferes with the generation of active caspase-8, attenuating the role of DISC.

(second mitochondria-derived activator of caspases)/DIABLO (direct inhibitor of apoptosis with low pi) which is released from mitochondria by Bax during apoptosis can antagonise their effect [21]. This role explains why Bax inactivation or mutation in mismatch-repair (MMR)deficient tumours can be responsible for TRAIL resistance [7, 22]. Also, Bax introduction in Bax deficient cells restored TRAIL sensitivity [21]. Recent studies also indicate that combination of TRAIL with chemotherapy or radiation can overcome TRAIL resistance by overexpression of Bax [7, 23-24].

Interestingly these TRAIL apoptotic effects tend to be more abundant in cancer cells compared to normal cells [25-27].

\section{TRAIL in oncology field}

\section{The promising cancer apoptotic inducer}

Multiple cancer cells have developed mechanisms to evade a tightly regulated cell death programme, rendering a more aggressive pattern to the disease. These mechanisms include inactivating proapoptotic cell death components or manipulating the levels of antiapoptotic molecules [28]. Thus, it is unsurprising that using naturally present apoptosis inducer molecules in cancer treatment has become a reasonable approach. Among these molecules used in induction of apoptosis is the Fas ligand [29] and members of TNF family [30]. When compared to other TNF family members and Fas ligand TRAIL showed more safety as TNF and Fas induce cytotoxicity against tumour cells with a lethal inflammatory response caused by the first and severe hepatotoxicity caused by the second in murine models [31]. The balance between TRAIL efficiency associated with selectivity to cancer cells and potential safety made it a powerful tool in cancer treatment [32-33]. Also, the molecular pathway of TRAIL spotted it as a promising apoptosis induction agent ahead of other chemotherapeutic formulations. The supremacy of TRAIL over most DNA-damaging drugs also lies in its ability to induce apoptosis in different cell lines regardless the p53 status [34]. These chemotherapeutics depend solely on p53 for intrinsic apoptosis pathway induction and hence the molecular pathway of TRAIL provides a solution circumventing tumour treatment resistance acquired through mutations in p53. Added to that, another attractive feature is that combination of chemotherapeutics and TRAIL lead to synergism in apoptosis induction [35]. Interestingly, it is to be noted that ionising radiation (IR), a mainstay regimen in many cancer types treatment, can sensitise cancer cells to TRAIL action as well [36]. This synergism has been attributed to many molecular and cell signalling changes [3, 24, 37].

This synergism can be used in a TRAIL combinational approach to reach maximum apoptosis in cancer. Another consequential benefit from this synergism is the usage of lower doses of either chemotherapeutics or radiation and hence limiting their well-known systemic side effects. Another additive feature of TRAIL over these agents is its ability to affect cancer stem cells. 


\section{TRAIL effects in eradicating cancer stem cells}

Cancer stem cells (CSCs), have been proposed to be responsible for the self-renewal, growth of tumours, and their recurrence after treatment owing to the analogy that maintenance of adult normal tissues is mediated through tissue-specific SCs [38-39]. Therefore the new era in cancer therapies lies in targeting and killing CSCs. Like other tumour cells, CSCs possess death receptors (DR) that can be targeted by TRAIL [40]. It was found that DR are expressed at a high level in glioblastoma and lung CSCs [41] and that the chemoresistance in colon CSCs was acquired through upregulation of DR4 [42]. These facts highlight TRAIL potential as a CSCs eliminating agent. However, in some situations where apoptotic signalling pathways are deregulated in CSCs, TRAIL-based combinational approaches are required. In CD133+ glioblastoma, T-cell acute leukaemia, and breast cancer cells, the TRAIL inhibitory cFLIPs proteins are upregulated [43]. Silencing of cFLIPs by siRNA and its downregulation by cisplatin restores cell sensitivity to death stimuli, suppressing CSC self-renewal and tumour metastasis [44-45].

Excitingly, the three musketeers of CSCs self-renewal; Wnt, Notch, and Hedgehog pathways can be knocked down using TRAIL-based combination leading to elimination of cancer recurrence and relapse.

In the Wnt pathway, the Wnt family secreted proteins bind to specific Frizzler (FZD) receptors on the surface of CSCs activating intracellular pathways, resulting in $\beta$-catenin protein uncoupling from its degradation complex and nuclear translocation [46] as depicted in Figure 2. This nuclear $\beta$-catenin binds to T-cell factor 4 (Tcf4) resulting in target gene activation, including cyclin D1, c-Myc, and survivin allowing apoptosis escape. Treatment of triple negative breast cancer (TNBC) stem cells with cisplatin and TRAIL results in inhibition of Wnt downstream target, $\beta$-catenin, phospho $\beta$-catenin, and cyclin D1 leading to increasing apoptosis and reduced proliferation [47].

Interference of Hedgehog pathway using genetic and pharmacological tools also sensitises cells to TRAIL cytotoxicity. The Hedgehog-GLI pathway modulates DR4 expression by binding of the transcription factor GLI3 to DR4 promoter. Hence targeted knock down of GLI-3 by small interfering RNA (siRNA) resulted in restoration of DR4 expression and TRAIL sensitivity [48]. Using cyclopamine, a well-established pharmacological inhibitor of smoothened (SMO) which is the signalling component of the Hedgehog receptor complex, lead to sensitisation of cholangiocarcinoma cells to TRAIL [49]. Cyclopamine treatment downregulated cellular IAP-1 (clAP-1) and X-linked IAP (XIAP) protein levels via the Hedgehog inhibition dependent mechanism. Also, the same study concluded that Hedgehog inhibition can promote TRAIL sensitisation independently from Bid, Bim, Bax, and Bak.

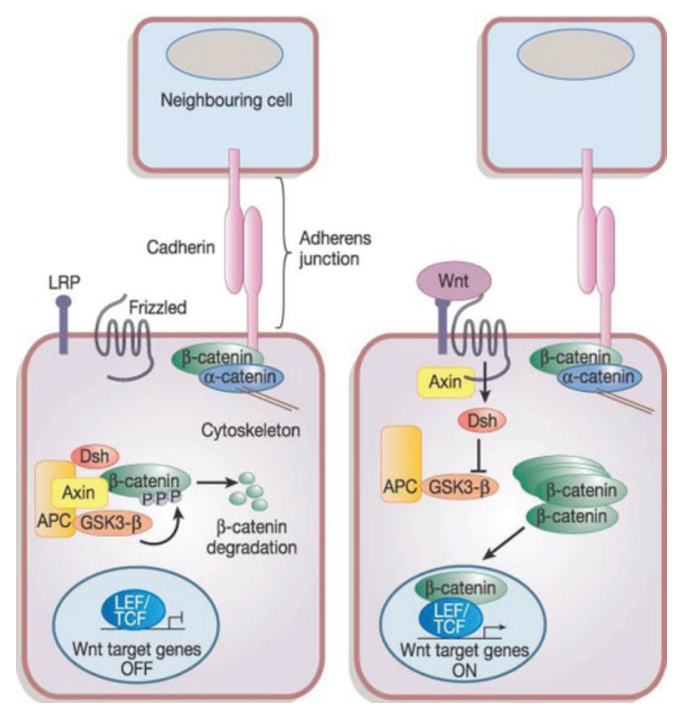

Figure 2. The canonical Wnt signalling pathway. Reused with permission of [121]. 
Notch is a transmembrane heterodimeric receptor present in four different forms in humans (Notch 1-4) [50]. Since all Notch receptors are activated by $\gamma$-secretase using inhibitors of this enzyme (GSIs) with TRAIL lead to sensitisation MDA-MB-231 breast cancer cells to TRAIL induced apoptosis [51]. The underlying mechanism for that synergism lies in Notch inhibition by GSIs leading to: 1) transcriptional activation of DR4 and DR5 and their overexpression via AP1 dependent mechanism, 2) downregulation of survival factors as phosphorylated form of AKT and IAP members like survivin and C-IAP-2 and 3) increase in proapoptotic elements as Noxa and members of the Bcl-2 family.

However, the complete relation between self-renewal pathways and TRAIL signalling in different types of CSCs remains a little bit ambiguous and require future analysis for better understanding.

Based on these promising effects as apoptosis inducer, scientists introduced soluble human recombinant TRAIL and agonistic monoclonal antibodies targeting either DR4 or DR5 in clinical fields [52].

\section{TRAIL in clinical trials}

In general most TRAIL preparations have passed phase I trials with no significant toxicity and were tolerated by most of the patients. However, a literature review of phase II trials up till now shows that different TRAIL preparations did not meet the expectations and had nearly no impact when used as monotherapy or in addition to standard regimens.

A randomised phase II study was done by Soria et al to evaluate the efficacy and safety of dulanermin(soluble recombinant human TRAIL) combined with paclitaxel and carboplatin (PC) and bevacizumab(B) as first-line treatment for advanced or recurrent non-small-cell lung cancer [53]. The addition of dulanermin to PC and PCB had no impact on progression-free survival (PFS) and overall survival (OS).

Tigatuzumab (CS-1008) is so far the most widely assessed monoclonal antibody targeting TRAIL-R2. In 2013 a randomised phase II study was performed using tigatuzumab versus placebo as a combination therapy with carboplatin and paclitaxel in patient with advanced NSCLC, tigatuzumab did not improve the efficacy of carboplatin/paclitaxel treatment [54].

Tigastuzumab was also tested in combination with gemcitabine for treatment of metastatic pancreatic cancer, it was well tolerated during the trial and the numeric trends described in the study suggested a possible contribution of tigatuzumab to the antitumour efficacy of gemcitabine. However there were no definitive conclusions which can be drawn regarding the contribution of tigatuzumab to the observed overall response rate (ORR), PFS, or overall survival data (OSD) [55].

Mapatumumab, also called TRM1 or HGS-ETR1, is the only TRAIL-R1 antibody assessed in clinical trials. Monotherapy trials assessed its role against advanced solid tumours like refractory non Hodgkin lymphoma [56], refractory colorectal cancer [57], and stage IIIb/IV refractory non small cell lung cancer [58]. Mapatumumab was well tolerated in general, however, the results were disappointing as none of these approaches have significantly impacted the OS of patients.

Conatumumab, a humanised monoclonal agonistic antibody against TRAIL-R2, was assessed in combination with FOLFIRI chemotherapy as a second-line treatment to patients with mutant KRAS metastatic colorectal carcinoma. It showed a trend toward improvement of PFS with tolerated toxicity [59]. Another trial was done by Fuchs et al in which conatumunab was tested against placebo as a combination therapy with mFOLFOX6 plus bevacizumab in treatment of metastatic colorectal cancer. In contrast to a former study conatumumab did not demonstrate improved efficacy over placebo [60].

The failure of TRAIL preparations in proving a statistical benefit in OS or PFS might be attributed to pharmacokinetic properties of such preparations or inability to reach proper concentration of preparation at site of action.

Another proposed mechanism is the development of TRAIL resistance in cancer cells by one or more of the mechanisms mentioned previously.

TRAIL gene transfer appear to be a promising alternative as it results in the production of targeted stable high concentration in cancer tissues and possible bystander effects [61]. Also, the role of cancer gene therapy has been increasing specially after (T-Vec) approval by the FDA in 2015 for melanoma treatment [62]. In the following section we intend to review all the methods used for TRAIL gene delivery. 


\title{
TRAIL gene delivery system
}

\author{
Viral vectors for TRAIL gene delivery
}

Viral vectors demonstrated more privileges over non-viral ones because of their natural efficient cell attachment abilities, entry, and highest level of transgene expression as part of the viral replication cycle [63-64].

These viral vectors are derived from adenoviruses, adeno-associated viruses, retroviruses, poxviruses, and herpesviruses with each and every one of them having its own advantages and limitations. Adenoviruses because of their relatively simple production and manipulation, high transduction efficacy without being integrated in cell genome, and ability to accommodate up to 30-kb DNA are preferred over other viral vectors. Both helper-dependent high-capacity Ad vectors that completely lack viral coding sequences or conditionally replicating Ads (also known as CRAds) driven by tumour-specific promoters in their E1A regions have been used to deliver TRAIL gene [24, 61, 65]. CRAds are dependent on coxsackie-adenovirus receptor (CAR) for cell entry. However, they can also be engineered to target alternative cell receptors that are overexpressed in tumour tissues than in normal tissues like CD46 [66]. This fact makes adenoviruses a promising vector to overcome obstacles of viral gene transduction therapy in terms of cell entry and toxicity to normal tissues. The ability of Ad vector to transfer full length of TRAIL cDNA selectively to tumour cells resulted not only in its rapid production and cancer apoptosis, but it also had bystander effect which is believed to augment its therapeutic and clinical value [67]. In the same experiment Seol et al [67], assumed that Ad TRAIL bystander effect is media transferable not contact mediated as demonstrated by Kagawa et al [61]. However, both effects are very useful in clinical situations as they can overcome any viral limited transduction efficiency in tumours. It is also to be noted that intracellular pathway of apoptosis induced by Ad-TRAIL differs from its media transferable-bystander effect where it is released passively from dying cells to interact with neighbouring cells receptors.

Another advantage of these adenoviral vectors is that they can be double armed with any gene beside the one coding TRAIL to potentiate the tumoural cytotoxicity of the cancer targeted gene virotherapy (CTGVT) or to overcome TRAIL resistance. Reconstruction and recombination of two different adenoviral vectors one carrying shRNA and the other coding TRAIL in E.coli cells resulted in single double armed vector expressing shRNA beside TRAIL [68-69]. These short hairpin RNA that can interfere with cancer cells RNA can be used to silence the expression of anti-apoptotic proteins like the IAP family member survivin [68], the nuclear protein Daxx [69], or the $\beta$-galactoside binding protein galectin-1 [70] and thereby augment the cytotoxic effect of TRAIL in cancer cells. CRAd coding TRAIL can also be loaded and double armed with a separate cassette coding Arresten gene. This dual expression can also potentiate the TRAIL induced cancer cell apoptosis as Arresten affects different cell signalling pathways to inhibit angiogenesis and tumour growth [66]. Also, double armed vectors loaded with suppressor of cytokine signalling 3 (SOCS3) has shown to reverse the resistance of HCC to TRAIL-induced apoptosis through the interaction between SOCS3 and JAK/STAT pathway [71].

Another virus that has not been extensively studied as adenovirus for TRAIL delivery is the Newcastle disease virus (NDV). Recombinant NDV have shown oncolytic effects either alone or in combination to other genes or drugs in many pre-clinical studies [72-73] and is being considered for clinical trials now. The rNDV expressing TRAIL gene and IL-2 was found to induce apoptosis in mouse models bearing hepatocellular carcinoma and malignant melanoma successfully [74]. In late 2015, a study showed that not only TRAIL can be transmitted by rNDV but also can be used synergistically to augment the oncolytic effect of this virus as caspase-8 activated byTRAIL binding to DR4/ DR5 amplifies the intrinsic mitochondrial cycle initiated by rNDV [73].

\section{Non viral vectors for TRAIL gene delivery}

Non-viral systems for gene transfer include deliberately introducing nucleic acids into cells DNA using nanotechnology, mesenchymal cells, microparticles. The chemical methods include a variety of liposomes, peptide delivery systems, cationic polymers causing disruption of the cell membrane. Physical methods for such include electroporation or ultrasonography.

Liposomes with bioactive TRAIL tethered to their surfaces did not only act as an efficient mode of its delivery, but rather improved its cytotoxicity by clustering of DR5 on cell surface [75]. Also, cationic liposomes can be modified and engineered to act as co-delivery system double like adenoviruses to express human TRAIL gene beside other drugs to improve tumour cells apoptosis outcome. This concept was tested using liposomes double delivering TRAIL and paclitaxel in glioma and found to be an effective method of either in vivo or in vitro dual delivery [76]. 
The role of cationic polymers is not only restricted to TRAIL gene intra-tumoural delivery but can also be used to enhance the transfection of TRAIL encoding adenoviruses in cells that down express (CAR) by overcoming the repulsion between the epithelial cells coated with anionic glycosaminoglycan and negatively charged adenoviral surfaces [77]. The ethylene glycol diglycidyl ether (EGDE)-3,3'-diamino-Nmethyl dipropylamine $\left(3,3^{\prime}\right)$ also coined as (EGDE-3,3') polymer is a promising tool in this regard as it lacks the toxic effects of the most commonly used polymer polyethylenimine $(\mathrm{pEI})$ and enhances the transduction of Ad TRAIL in bladder cancer cells lacking (CAR) [78].

The peptide delivery system implies the delivery of targeted genes or protein in living cells using a protein transduction domain (PTD) or cell penetrating peptides [79]. The number of available PTD is enormous and keeps growing since the region between amino acids $47-57$ of the transactivator of transcription (TAT) protein of HIV-1 (human immunodeficiency virus type 1) was found to be responsible for cellular membrane penetration [80]. Arginine-rich intracellular delivery (AID) peptides were found to be successful in delivering plasmids coding human TRAIL into human lung carcinoma A549 cells [81]. Also, peptides can be used for targeted gene delivery by binding to specific integrins only expressed in cancer cells or tumour blood vessels. In this regard, ACDCRGDCFC a targeting peptide to human $\alpha_{v} \beta_{3}$ and $\alpha_{v} \beta_{5}$ on tumour blood vessels was fused to TRAIL forming what is called RGD-L-TRAIL and improved the therapeutic index of TRAIL in vitro and in vivo [82].

Chimeric polypeptides formed by cross-linkage of octa-D-arginine (cell penetration-facilitating group and DNA-binding moiety) and tetraL-histidine (for the acquired endosomal buffering capacity of the polypeptide) are reported to be highly efficient vectors for TRAIL delivery.

TRAIL delivered by reducible chimeric polypeptides considerably inhibited tumour growth formation in mice xenografted with HeLa cells [83].

Microparticles formed of biodegradable polymers are also another effective mode for TRAIL delivery. PEGylated TRAIL microparticles (where Mono-methoxy PEG (mPEG) molecule is added to the microparticle) showed supremacy to regular TRAIL microparticles in terms of encapsulation efficacy and low initial burst release. In vitro experiments showed $15.8 \%$ calculated burst release from PEG-TRAIL microspheres in comparison to $42.7 \%$ for TRAIL microspheres. Also, in vivo experiment showed $25.9 \%$ and $78.3 \%$ tumour regression for TRAIL microparticles and PEG-TRAIL respectively at $300 \mu \mathrm{g}$ of TRAIL/mouse [84] as depicted in Figure 3.

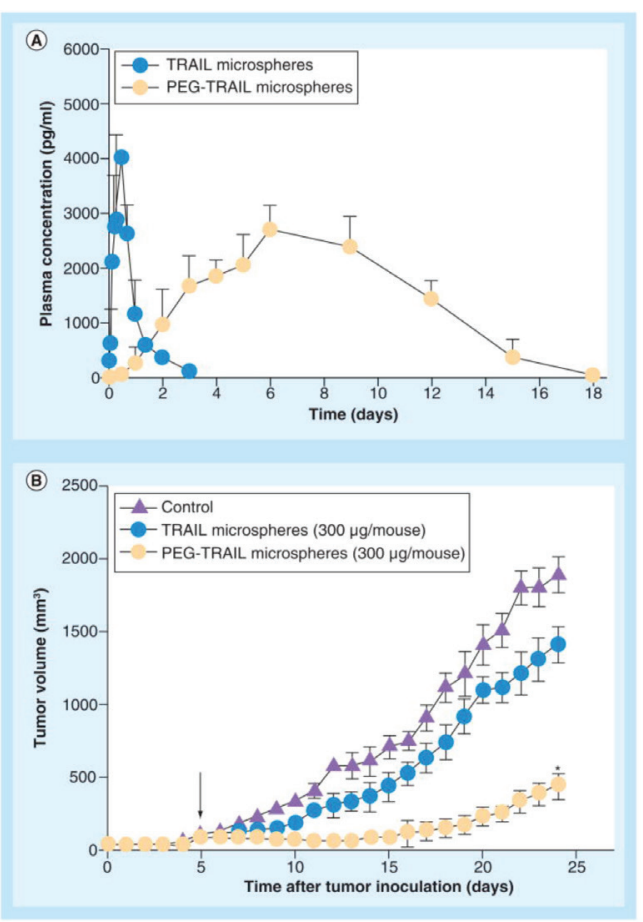

Figure 3. (A) In vivo pharmacokinetic profiles of TRAIL or PEG-TRAIL microspheres after subcutaneous administration (100 $\mu$ g/rat; $n=5$ ); (B) Tumour growth suppressions by TRAIL or PEG-TRAIL microspheres (300 $\mathrm{\mu g} /$ mouse, subcutaneous). Reused use permission of [122]. 
Synthetic polyurethanes (PUs) which have the ability to incorporate urethane (carbamate) bonds into polymeric backbone were used as delivery systems as well. Paclitaxel loaded water borne polyurethane nanomicelles conjugated with TRAIL (PTX-PU-TRAIL) have been tested for efficacy against $4 \mathrm{~T} 1$ bearing balb/c mouse model [85].

Transdifferentiation (TD)-derived induced neural stem cells (iNSCs) have been engineered with an image able fusion between TRAIL (iNSC-diTR) and Gaussia luciferase [86]. Results revealed that treating LN18 and U87 glioblastoma cell lines with conditioned media from iNSC-sTR treatment considerably enhanced activity of caspase-3/7 in a time dependent manner. Furthermore, in the treatment of mC-FL expressing U87 glioblastoma cells intra-cranially implanted in mice, the survival rate of mice treated with iNSC-sTR induced tumour regression and survival increase.

\section{Mesenchymal cells for TRAIL gene transfer}

Mesenchymal cells (MSCs) can be isolated from either adult or fetal tissues. Their ability to migrate to tumour sites as well as given their pre-metastatic niche, low immunogenicity, easy reproduction, genetic manipulation, and modification make them a very promising tool for transgene expression [87]. These facts caused them to be extensively studied in comparison to other non-viral gene delivery systems for TRAIL delivery. However, it is to be noted that each tumour type treatment requires a specific MSCs type to achieve the desired results. This fact was concluded after Akimoto et al discovered that co-culturing adipose tissue derived MSCs (AT-MSCs) with GBM cells induced tumour vascularisation and proliferation, while co-culturing with human umbilical cord blood-derived MSCs (HUMCs) inhibited tumour growth [88]. Also, the mode of delivery of MSCs harbouring TRAIL (MSCs-TRAIL) affected their tumour incorporation as it is seen that IV delivered MSCs incorporated more in tumours than intrapleurally-delivered MSCs [89].

Virally transfected or non-viral gene modifiedMSCs encoding soluble TRAIL were tested against different types of tumours. It was concluded that AT-MSCs-TRAIL were effective against sarcomas by inducing apoptosis in different osteosarcoma, Ewing's Sarcoma (ES), and rhabdomyosarcoma cell lines in vitro and in pre-established ES xenotransplants in vivo [90]. Human malignant mesothelioma responded as well to the antitumour effects of TRAIL-expressing MSCs derived from human bone marrow, and the inflammatory tumour environment in vivo was successfully reduced after their administration [91]. An antitumour activity was also noted in TRAIL sensitive intracranially implanted medulloblastoma and in TRAIL-resistant MBs pretreated with MS-275 after human MSCs-TRAIL treatment [92]. Also, non-viral TRAIL-engineered AD-MSCs were capable to migrate towards multiple myeloma cells and induce tumour cytotoxic effects [93]. Also, MSCs transducing TRAIL were able to target a subgroup of cancer stem cells resistant to common oncological treatments known as side population (SP) [94].

Engineered human umbilical cord mesenchymal stem cells (HUMSCs) to secrete soluble TRAIL via adenoviral transduction mediated by cellpermeable peptides were found to be very effective in reduction of glioma in in-vitro cultures and also to migrate efficiently towards human glioma cells and prolong the survival of athymic nude mouse bearing intracranial xenografts of human glioma [95]. Also, Ad.sTRAIL(DR4) transfected MSCs exhibited tumour inhibition against pancreatic cancer cells PancTu1, while colorectal cancer cells HCT116 responded to MSCs transfected with Ad.sTRAIL(DR5) [96]. Adenovirus was not the only virus that was used to transfect MSCs. The human MSCs transduced with a lentiviral vector encoding TRAIL successfully migrated to malignant mesothelioma [5] and to hepatocarcinoma driven by AFP promoter [97] and induced tumour cells apoptosis. Also, rat bone marrow MSCs encoding S TRAIL via lentiviral vector transduction were able to induce hepatocarcinoma growth inhibition in mouse models [98]. Using lentivirus-TRAIL, transduced MSCs induced significant apoptosis in different types of cancer cell lines as well as showed remarkable reduction in metastatic tumour burden with frequent eradication of metastasis in murine lung metastasis mode [99]. Interestingly, herpes simplex virus (HSV) type I amplicon vector secreting the trimeric form of TRAIL was used to engineer MSCs and showed increased survival of mouse models with intracranial glioma [100]. It is to be noted that combinational approaches with different chemotherapeutic agents as cisplatin [101], lipoxygenase inhibitor MK886 [102], and temozolomide [103] enhanced the apoptotic effect of MSCs-TRAIL against different cancer types.

Recently a new role for MSCs has been explored. Kaczorowski et al, used MSCs isolated from bone marrow as delivery vectors for oncolytic adenovirus (OAd5/3-TRAIL). They proved that this method of viral delivery was more efficient and tumour selective than the virus alone and had a tendency towards cancer stem cells targeting [104].

Knowing the selective MSCs homing ability to specific types of cancer cells can subsequently improve TRAIL delivery and apoptosis induction. Intravenously injected HUMSCs have been shown to migrate to lung cancer after 24 hours via MCP-1/CCR2 transduction cascade [30]. This 
cascade consists of overexpression of MCP-1 on lung cancer A549 cells and expression of its receptor CCR2 on the surface of HUMSCs. These TRAIL-expressing HUMSCs dramatically enhanced apoptotic rate in tumour cells and prolonged survival of xenografted mice.

\section{Nanotechnology}

Recently,different modalities of colloidal and non-colloidal nanoparticles (NPs) with different biological properties and compositions have been investigated for drug/gene delivery in cancer therapy as depicted in Table 1. Nanoparticles are taken up by cells more efficiently than larger micromolecules and therefore could be used as effective transport and delivery systems. Nanovectors in comparison with free antitumour drugs showed better therapeutic results and enhanced intra-tumoural accumulation [105] as depicted in Figure 4. Their effectiveness is attributed to their small size allowing penetration of cell membranes, proteins stabilisation, and binding and escaping lysosomal degradation after endocytosis.

Table 1. Different types of nanoparticles investigated for drug/gene delivery.

\begin{tabular}{|l|l|l|}
\hline \multicolumn{1}{|c|}{ Particle class } & \multicolumn{1}{|c|}{ Materials } & \multicolumn{1}{c|}{ Application } \\
\hline Natural materials or derivatives & $\begin{array}{l}\text { Liposomes } \\
\text { Chitosan } \\
\text { Gelatine } \\
\text { Dextrane } \\
\text { Starch } \\
\text { Alginates }\end{array}$ & Drug/gene delivery \\
\hline Dendrimers & Branched polymers & $\begin{array}{l}\text { Drug delivery/gene } \\
\text { delivery }\end{array}$ \\
\hline Polymer carriers & $\begin{array}{l}\text { Block copolymers } \\
\text { Polylactic acid } \\
\text { Polycaprolactone } \\
\text { Polyethyleinemine } \\
\text { Poly(cyano)acrylates }\end{array}$ & Drug/gene delivery \\
\hline Various & $\begin{array}{l}\text { Silica-nanoparticles } \\
\text { Mixtures of above }\end{array}$ & Gene delivery \\
\hline
\end{tabular}

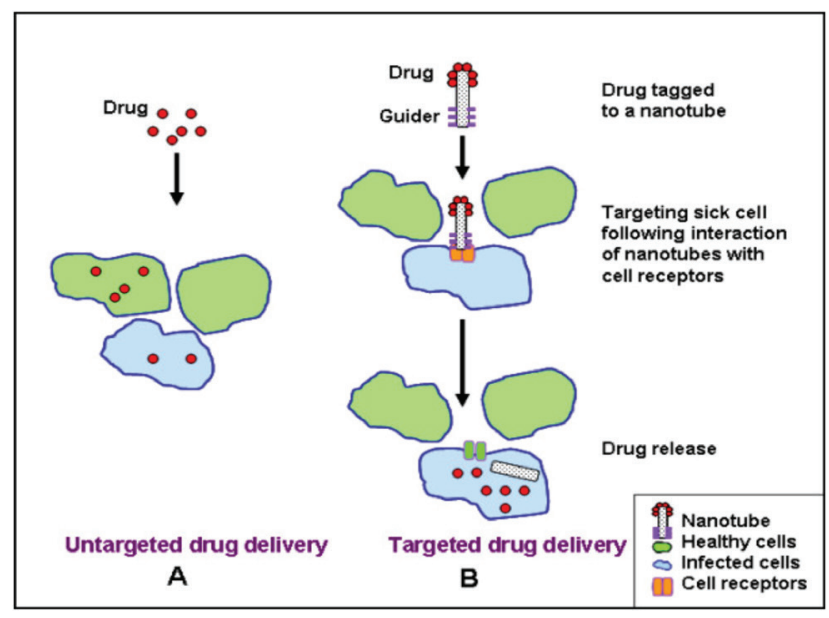

Figure 4. Comparison between untargeted and targeted drug delivery using a NP. 
Also, these nanosystems showed:

1 Passive accumulation into tumour target sites through a phenomena known as enhanced permeability and retention effect (EPR) because of lack of functional lymphatics and tumour angiogenesis [106].

2 Ability of targeted delivery by binding to specific cell receptors when modified with a guider ligand thus sparing the normal tissues and enhancing the drug/gene therapeutic index [106].

3 Modification of drug pharmacokinetics and biological distribution [107].

4 Prolonged presence in the bloodstream when their surface is coated with polyethylene glycol (PEG) molecule which helps escaping phagocytosis by the reticuloendothelial system cells [108].

All these characteristics made nanovectors a very promising vector for targeted gene therapy and efficient TRAIL delivery was achieved using different nanotechnology-based systems.

A NP with an iron oxide core surrounded by a layer of chitosan-polyethylene glycol (PEG)-grafted polyethyleneimine (PEI) copolymer (CP-PEI) and modified with chlorotoxin (CTX) for glioblastoma targeting,successfully delivered plasmid coding human TRAIL to human T98G GBM cells. Moreover to in vitro increased apoptosis, complete regression of T98G-derived flank xenografts in nude athemic mice was reported after NP-TRAIL-CTX treatment [109].

Also, magnetic ferric oxide nanoparticles (NPs) conjugated withTRAIL demonstrated apoptotic activity against different human glioma cells and germline stem cells (GSCs). A targeting ability to U251 cell-derived glioma xenografts associated with tumour volume decrease and increased survival of animal models was also reported with these NPs [110].

A dendrimer, which is a nanosized polymer formed from a central core surrounded by successively added layers of branching structures called dendrons, has been used for transgene expression. An engineered triazine-which binds DNA through hydrogen bonds-modified on a polyamidoamine (PAMAM) dendrimer was used successfully to deliver TRAIL coding plasmid to osteosarcoma cell line MG-63. It also showed beside apoptosis induction low cytotoxicity and high transfection efficacy in comparison to other polymers [111]. These dendrimers do not only serve as transmission vectors but also can be used for targeted gene delivery. Transferrin (Tf)-modified (PAMAM) dendrimer which has great brain targeting efficiency [112] was used to deliver (hTRAIL)-encoding plasmid in C6 cells and showed enhanced cytotoxicity, glioma targeting, and enhanced permeability and retention (EPR) effect in comparison to non modified NPs [113].

Interestingly, the NPs can be also designed to act as a dual delivery system making them an optimum vector paving the path to optimum TRAIL gene-based therapies. The advantage of any chemotheraputic combination with TRAIL does not only aim to enhance its cytototoxic effects, but rather to achieve better safety profiles by dose reduction resulting from combination synergism.

A polylactic-co-glycolic acid (PLGA)-based multi-layered nanoparticles (MLNP) was used as co-delivery system for camptothecin (CPT) a topoisomerase-1 inhibitor and plasmid coding rhTRAIL. Beside the enhancend in vitro and in vivo apoptosis in different cell lines treated by these MLNPs, this co-delivery system succseded in reducing the CPT and pTRAIL dosing by 3.1-15 fold and 4.7-8.0 fold respectively because of their synergestic action [114].

Another design of NPs that can serve as one pot, delivering combinational formulation of genes and chemotheraputic agents, is the albumin-bound nanoparticle (HSA-NP). Pancreatic cancer Mia Paca-2 cells showed apoptosis following treatment with HSA-NP delivering paclitaxel and TRAIL [115] while HCT116 coloncancer cell were inhibited by HSA-NP carrying doxorubicine and TRAIL [116].

In accordance with the approach of drug and gene co-delivery via cationic liposomes, nanoscale designed liposomes were used to deliver doxorubicin and TRAIL to produce synergistic apoptotic effct in non-small cell lung cancer (NSLC) [117] and glioblastoma multiforme (GBM) cancer cells [118]. Intrestingly,double-genetherapy for coloncancer was mediated by a nanoliposome containing tyrosinekinase receptor 3 ligand and TRAIL-encoding plasmids [119]. This double design offers a new route to combined gene therapy and immunotherapy for cancer treatment.

There is a recent report highlighting significance of transformable core-shell-based nanocarrier (CS-NG) as an effective system to deliver TRAIL and anti-angiogenic agents [120]. CS-NG enzymatically assembled into micro-sized extracellular depots at the site of tumour with the help of an enzyme hyaluronidase, frequently overexpressed in the tumour microenvironment. Structurally, CS-NG is composed of coreNG (C-NG) loaded with therapeutics, shell-NG (S-NG) with encapsulated transglutaminase (TG), and human serum albumin (HSA), coated on the surface of C-NG. Acid-degradable polymers for delivery of therapeutically effective agents have attracted considerable attraction. 
CS-NG is equipped with an acid-degradable modality, and therefore it effectively releases combinatorially delivered cilengitide (antiangiogenic agent) and TRAIL to endothelial cells at the acidic tumour microenvironment and membrane of cancer cells respectively. This method showed effectiveness against breast cancer MDA-MB-231 cells.

\section{Conclusion}

Induction of apoptosis could be the ultimate approach for different types of cancer treatment. TRAIL is a powerful molecular agent for apoptosis induction. The recent approval of gene therapy in the form of (T-Vec) for melanoma treatment paves the pathway towards succesful future gene therapy trials. Incorporating TRAILin future oncolytic viruses, based on its selective potency and interaction with CSCs, will definitely enhance their outcome. Using hybrid gene delivery systems, a safe viral vector targeting cellular integrins and receptors of CSCs through peptide targeting ability can be achievied. Other options for promising gene therapy clinical trials approaches will include TRAIL transgene delivery by mesenchymal stem cells and nanoparticles. Likewise, combining TRAIL with chimeric antigen receptor (CAR) T-cell, could augment the effect of this approach. To this end, TRAIL usage could tackle a myriad of oncological conditions thereby achieving a shift in the paradigm of cancer treatment.

\section{Acknowledgments}

This work was carried out as part of a collaboration between Alexandria University, Egypt and the University of Alabama at Birmingham, USA with the help of Professor Mona Fouad, MPH. We would like to thank Mostafa Samir (Alexandria Comprehensive Cancer Center) Baher Ibrahim, MD, MS (Kings College London) and Sally Abdlemalek (St George University of the Caribbean) for their help during the administrative process and logistic preparation of this article.

\section{References}

1. Kerr JF, Wyllie AH, and Currie AR (1972) Apoptosis: a basic biological phenomenon with wide-ranging implications in tissue kinetics Br J Cancer 26(4) 239-57 DOI: 10.1038/bjc.1972.33 PMID: 4561027 PMCID: 2008650

2. Cotter TG (2009) Apoptosis and cancer: the genesis of a research field Nat Rev Cancer 9(7) 501-7 DOI: 10.1038/nrc2663 PMID: $\underline{19550425}$

3. Koff JL, Ramachandiran S and Bernal-Mizrachi L (2015) A time to kill: targeting apoptosis in cancer Int J Mol Sci 16(2) 2942-55 DOI: 10.3390/ijms16022942 PMID: 25636036 PMCID: 4346874

4. Wiley SR et al (1995) Identification and characterization of a new member of the TNF family that induces apoptosis Immunity 3(6) 673-82 DOI: 10.1016/1074-7613(95)90057-8 PMID: $\underline{8777713}$

5. Pitti RM et al (1996) Induction of apoptosis by Apo-2 ligand, a new member of the tumor necrosis factor cytokine family $J$ Biol Chem 271(22) 12687-90 DOI: $\underline{10.1074 / j b c .271 .22 .12687}$ PMID: $\underline{8663110}$

6. Ivanov VN, Bhoumik A and Ronai Z (2003) Death receptors and melanoma resistance to apoptosis Oncogene 22(20) 3152-61 DOI: $10.1038 /$ sj.onc.1206456 PMID: 12789291

7. LeBlanc $\mathrm{H}$ et al (2002) Tumor-cell resistance to death receptor--induced apoptosis through mutational inactivation of the proapoptotic Bcl-2 homolog Bax Nat Med 8(3) 274-81 DOI: 10.1038/nm0302-274 PMID: 11875499

8. Ozoren N and El-Deiry WS (2003) Cell surface death receptor signaling in normal and cancer cells Semin Cancer Biol 13(2) 135-47 DOI: 10.1016/S1044-579X(02)00131-1 PMID: 12654257

9. Pan G et al (1997) An antagonist decoy receptor and a death domain-containing receptor for TRAIL Science 277(5327) 815-8 DOI: $10.1126 /$ science.277.5327.815 PMID: $\underline{9242610}$ 
10. Degli-Esposti MA et al (1997) The novel receptor TRAIL-R4 induces NF-kappaB and protects against TRAIL-mediated apoptosis, yet retains an incomplete death domain Immunity 7(6) 813-20 DOI: 10.1016/S1074-7613(00)80399-4

11. Walczak $\mathrm{H}$ et al (1997) TRAIL-R2: a novel apoptosis-mediating receptor for TRAIL EMBO J 16(17) 5386-97 DOI: $10.1093 /$ emboj/16.17.5386 PMID: $\underline{9311998}$ PMCID: 1170170

12. Pan G et al (1997) The receptor for the cytotoxic ligand TRAIL Science 276(5309) 111-3. DOI: 10.1126/science.276.5309.111 PMID: 9082980

13. Marsters SA et al (1997) A novel receptor for Apo2L/TRAIL contains a truncated death domain Curr Biol 7(12) 1003-6 DOI: 10.1016/S0960-9822(06)00422-2

14. Wu GS et al (1997) KILLER/DR5 is a DNA damage-inducible p53-regulated death receptor gene Nat Genet 17(2) 141-3 DOI: 10.1038/ng1097-141 PMID: $\underline{9326928}$

15. Sheridan JP et al (1997) Control of TRAIL-induced apoptosis by a family of signaling and decoy receptors Science 277(5327) 818-21 DOI: $10.1126 /$ science.277.5327.818 PMID: $\underline{9242611}$

16. Ashkenazi A (2002) Targeting death and decoy receptors of the tumour-necrosis factor superfamily Nat Rev Cancer 2(6) 420-30 DOI: $10.1038 / n r c 821$ PMID: 12189384

17. Mongkolsapaya $\mathrm{J}$ et al (1999) Structure of the TRAIL-DR5 complex reveals mechanisms conferring specificity in apoptotic initiation Nat Struct Biol 6(11) 1048-53 DOI: 10.1038/14935 PMID: 10542098

18. Hymowitz SG et al (1999) Triggering cell death: the crystal structure of Apo2L/TRAIL in a complex with death receptor $5 \mathrm{Mol}$ Cell 4(4) 563-71 DOI: 10.1016/S1097-2765(00)80207-5 PMID: 10549288

19. Green DR (2000) Apoptotic pathways: paper wraps stone blunts scissors Cell 102(1) 1-4 DOI: 10.1016/S0092-8674(00)00003-9 PMID: 10929706

20. Fulda $\mathrm{S}$ et al (2001) Cell type specific involvement of death receptor and mitochondrial pathways in drug-induced apoptosis Oncogene 20(9) 1063-75 DOI: 10.1038/sj.onc.1204141 PMID: 11314043

21. Deng $Y$, Lin $Y$ and Wu $X$ (2002) TRAIL-induced apoptosis requires Bax-dependent mitochondrial release of Smac/DIABLO Genes Dev 16(1) 33-45 DOI: 10.1101/gad.949602 PMID: 11782443 PMCID: 155309

22. Burns TF and El-Deiry WS (2001) Identification of inhibitors of TRAIL-induced death (ITIDs) in the TRAIL-sensitive colon carcinoma cell line SW480 using a genetic approach J Biol Chem 276(41) 37879-86 PMID: 11486001

23. Wang S and El-Deiry WS (2003) Requirement of p53 targets in chemosensitization of colonic carcinoma to death ligand therapy Proc Natl Acad Sci U S A 100(25) 15095-100 DOI: 10.1073/pnas.2435285100 PMID: 14645705 PMCID: 299914

24. Arafat $\mathrm{W}$ et al (2015) Targeted radiotherapy potentiates the cytotoxicity of a novel anti-human DR5 monoclonal antibody and the adenovirus encoding soluble TRAIL in prostate cancer J Egypt Natl Canc Inst 27(4) 205-15 DOI: 10.1016/j.jnci.2015.08.003 PMID: 26385392

25. Zhang XD et al (1999) Relation of TNF-related apoptosis-inducing ligand (TRAIL) receptor and FLICE-inhibitory protein expression to TRAIL-induced apoptosis of melanoma Cancer Res 59(11) 2747-53 PMID: 10364001

26. Chaudhary PM et al (1997) Death receptor 5, a new member of the TNFR family, and DR4 induce FADD-dependent apoptosis and activate the NF-kappaB pathway. Immunity 7(6) 821-30 DOI: $10.1016 / S 1074-7613(00) 80400-8$

27. Gura T (1997) How TRAIL kills cancer cells, but not normal cells Science 277(5327) 768 DOI: 10.1126/science.277.5327.768 PMID: $\underline{9273698}$

28. Igney FH and Krammer PH (2002) Death and anti-death: tumour resistance to apoptosis Nat Rev Cancer 2(4) 277-88 DOI: 10.1038/nrc776 PMID: 12001989

29. Linkermann $A$ et al (2005) Considering Fas ligand as a target for therapy Expert Opin Ther Targets 9(1) 119-34 DOI: 10.1517/14728222.9.1.119 PMID: 15757486 
30. Kiraz Y et al (2016) Major apoptotic mechanisms and genes involved in apoptosis Tumour Biol DOI: 10.1007/s13277-016-5035-9 PMID: 27059734

31. Imao $\mathrm{M}$ et al (2006) Differential caspase-9-dependent signaling pathway between tumor necrosis factor receptor- and Fasmediated hepatocyte apoptosis in mice Liver Int 26(1) 137-46 DOI: 10.1111/j.1478-3231.2005.01195.x PMID: 16420519

32. Ashkenazi A et al (1999) Safety and antitumor activity of recombinant soluble Apo2 ligand J Clin Invest 104(2) 155-62 DOI: 10.1172/JCI6926 PMID: 10411544 PMCID: $\underline{408479}$

33. Nesterov A et al (2004) Oncogenic Ras sensitizes normal human cells to tumor necrosis factor-alpha-related apoptosis-inducing ligand-induced apoptosis Cancer Res 64(11) 3922-7 DOI: 10.1158/0008-5472.CAN-03-2219 PMID: 15173003

34. Wang S and El-Deiry WS (2003) TRAIL and apoptosis induction by TNF-family death receptors Oncogene 22(53) 8628-33 DOI: 10.1038/sj.onc.1207232 PMID: 14634624

35. Nagane M, Huang HJ and Cavenee WK (2001) The potential of TRAIL for cancer chemotherapy Apoptosis 6(3) 191-7 DOI: 10.1023/A:1011336726649 PMID: 11388668

36. Silva MF et al (2014) lonizing radiations induce apoptosis in TRAIL resistant cancer cells: in vivo and in vitro analysis Asian Pac J Cancer Prev 15(5) 1905-7 DOI: 10.7314/APJCP.2014.15.5.1905 PMID: 24716909

37. Nagane M, Cavenee WK and Shiokawa Y (2007) Synergistic cytotoxicity through the activation of multiple apoptosis pathways in human glioma cells induced by combined treatment with ionizing radiation and tumor necrosis factor-related apoptosisinducing ligand J Neurosurg 106(3) 407-16 DOI: $\underline{10.3171 / j n s .2007 .106 .3 .407 ~ P M I D: ~} 17367063$

38. Visvader JE and Lindeman GJ (2008) Cancer stem cells in solid tumours: accumulating evidence and unresolved questions Nat Rev Cancer 8(10) 755-68 DOI: 10.1038/nrc2499 PMID: 18784658

39. Beck B and Blanpain C (2013) Unravelling cancer stem cell potential Nat Rev Cancer 13(10) 727-38 DOI: 10.1038/nrc3597 PMID: $\underline{24060864}$

40. He YC et al (2014) Apoptotic Death of Cancer Stem Cells for Cancer Therapy Int J Mol Sci 15(5) 8335-51 DOI: $10.3390 /$ ijms15058335 PMID: 24823879 PMCID: 4057734

41. Signore ML, Ricci-Vitiani L and De Maria R (2013) Targeting apoptosis pathways in cancer stem cells Cancer Lett 332(2) 374-82 DOI: $10.1016 / j . c a n l e t .2011 .01 .013$

42. Sussman RT et al (2007) Chemotherapy-resistant side-population of colon cancer cells has a higher sensitivity to TRAIL than the non-SP, a higher expression of c-Myc and TRAIL-receptor DR4 Cancer Biol Ther 6(9) 1490-5 DOI: 10.4161/cbt.6.9.4905 PMID: 17881904

43. Zobalova R et al (2008) CD133-positive cells are resistant to TRAIL due to up-regulation of FLIP Biochem Biophys Res Commun 373(4) 567-71 DOI: 10.1016/j.bbrc.2008.06.073 PMID: 18590703

44. Piggott $L$ et al (2011) Suppression of apoptosis inhibitor c-FLIP selectively eliminates breast cancer stem cell activity in response to the anti-cancer agent, TRAIL Breast Cancer Res 13(5) R88 DOI: 10.1186/bcr2945 PMID: 21914219 PMCID: 3262200

45. Ding $L$ et al (2011) Cisplatin restores TRAIL apoptotic pathway in glioblastoma-derived stem cells through up-regulation of DR5 and down-regulation of c-FLIP Cancer Invest 29(8) 511-20 DOI: 10.3109/07357907.2011.605412 PMID: 21877938 PMCID: $\underline{3255792}$

46. Reya T and Clevers $\mathrm{H}$ (2005) Wnt signalling in stem cells and cancer Nature 434(7035) 843-50 DOI: 10.1038/nature03319 PMID: 15829953

47. Yin S et al (2011) Cisplatin and TRAIL enhance breast cancer stem cell death Int J Oncol 39(4) 891-8 PMID: 21687939 PMCID: $\underline{3889134}$

48. Kurita S et al (2010) GLI3-dependent repression of DR4 mediates hedgehog antagonism of TRAIL-induced apoptosis Oncogene 29(34) 4848-58 DOI: 10.1038/onc.2010.235 PMID: 20562908 PMCID: 2928864 
49. Kurita $S$ et al (2011) Hedgehog inhibition promotes a switch from type II to type I cell death receptor signaling in cancer cells PLoS One 6(3) e18330 DOI: 10.1371/journal.pone.0018330 PMID: 21483830 PMCID: $\underline{3069071}$

50. Fortini ME (2009) Notch signaling: the core pathway and its posttranslational regulation Dev Cell 16(5) 633-47 DOI: 10.1016/j.devcel.2009.03.010 PMID: 19460341

51. Portanova P et al (2013) Notch inhibition restores TRAIL-mediated apoptosis via AP1-dependent upregulation of DR4 and DR5 TRAIL receptors in MDA-MB-231 breast cancer cells Int J Oncol 43(1) 121-30 PMID: 23686163

52. Micheau O, Shirley S and Dufour F (2013) Death receptors as targets in cancer Br J Pharmacol 169(8) 1723-44 DOI: $10.1111 /$ bph.12238 PMID: 23638798 PMCID: $\underline{3753832}$

53. Soria JC et al (2011) Randomized phase II study of dulanermin in combination with paclitaxel, carboplatin, and bevacizumab in advanced non-small-cell lung cancer J Clin Oncol 29(33) 4442-51 DOI: 10.1200/JCO.2011.37.2623 PMID: 22010015

54. Reck M et al (2013) A randomized, double-blind, placebo-controlled phase 2 study of tigatuzumab (CS-1008) in combination with carboplatin/paclitaxel in patients with chemotherapy-naive metastatic/unresectable non-small cell lung cancer Lung Cancer 82(3) 441-8 DOI: 10.1016/j.lungcan.2013.09.014 PMID: 24148258

55. Forero-Torres A et al (2013) Phase 2, multicenter, open-label study of tigatuzumab (CS-1008), a humanized monoclonal antibody targeting death receptor 5 , in combination with gemcitabine in chemotherapy-naive patients with unresectable or metastatic pancreatic cancer Cancer Med 2(6) 925-32 DOI: 10.1002/cam4.137

56. Younes $\mathrm{A}$ et al (2010) A Phase 1b/2 trial of mapatumumab in patients with relapsed/refractory non-Hodgkin's lymphoma $\mathrm{Br} J$

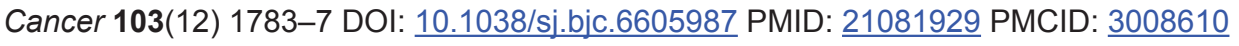

57. Trarbach T et al (2010) Phase II trial of mapatumumab, a fully human agonistic monoclonal antibody that targets and activates the tumour necrosis factor apoptosis-inducing ligand receptor-1 (TRAIL-R1), in patients with refractory colorectal cancer Br J Cancer 102(3) 506-12 DOI: 10.1038/sj.bjc.6605507 PMID: 20068564 PMCID: 2822942

58. Greco FA et al (2008) Phase 2 study of mapatumumab, a fully human agonistic monoclonal antibody which targets and activates the TRAIL receptor-1, in patients with advanced non-small cell lung cancer Lung Cancer 61(1) 82-90 DOI: 10.1016/j. lungcan.2007.12.011 PMID: $\underline{18255187}$

59. Cohn AL et al (2013) A randomized, placebo-controlled phase 2 study of ganitumab or conatumumab in combination with FOLFIRI for second-line treatment of mutant KRAS metastatic colorectal cancer Ann Oncol 24(7) 1777-85 DOI: 10.1093 / annonc/mdt057 PMID: 23510984

60. Fuchs CS (2013) et al TRAIL receptor agonist conatumumab with modified FOLFOX6 plus bevacizumab for first-line treatment of metastatic colorectal cancer: A randomized phase 1b/2 trial Cancer 119(24) 4290-8 DOI: 10.1002/cncr.28353 PMID: 24122767

61. Kagawa $S$ et al (2001) Antitumor activity and bystander effects of the tumor necrosis factor-related apoptosis-inducing ligand (TRAIL) gene Cancer Res 61(8) 3330-8 PMID: 11309289

62. Pol JG, Kroemer G and Galluzzi L (2016) First oncolytic virus approved for melanoma immunotherapy Oncoimmunology 5(1) p. e1115641 DOI: $\underline{10.1080 / 2162402 X .2015 .1115641}$

63. Cho DY et al (2013) Targeting cancer stem cells for treatment of glioblastoma multiforme Cell Transplant 22(4) 731-9 DOI: 10.3727/096368912X655136 PMID: 23594862

64. Ren $\mathrm{H}$ et al (2003) Immunogene therapy of recurrent glioblastoma multiforme with a liposomally encapsulated replicationincompetent Semliki forest virus vector carrying the human interleukin-12 gene-a phase I/II clinical protocol $J$ Neurooncol 64(1-2) 147-54 DOI: 10.1007/BF02700029 PMID: 12952295

65. Ye X et al (2005) Conditionally replicative adenovirus vector carrying TRAIL gene for enhanced oncolysis of human hepatocellular carcinoma Int J Mol Med 16(6) 1179-84 PMID: 16273304 
66. Li X et al (2012) A fiber chimeric CRAd vector Ad5/11-D24 double-armed with TRAIL and arresten for enhanced glioblastoma therapy Hum Gene Ther 23(6) 589-96 DOI: 10.1089/hum.2011.130

67. Seol JY et al (2003) Adenovirus-TRAIL can overcome TRAIL resistance and induce a bystander effect Cancer Gene Ther 10(7) 540-8 DOI: $\underline{10.1038 / \text { sj.cgt.7700597 PMID: } 12833134}$

68. Han Z et al (2015) Survivin silencing and TRAIL expression using oncolytic adenovirus increase anti-tumorigenic activity in gemcitabine-resistant pancreatic cancer cells Apoptosis 2015

69. Kang $S$ et al (2015) Silencing Daxx increases the anti-tumor activity of a TRAIL/shRNA Bcl-xL-expressing oncolytic adenovirus through enhanced viral replication and cellular arrest Cell Signal 27(6) 1214-24 DOI: 10.1016/j.cellsig.2015.02.028 PMID: $\underline{25748050}$

70. Li J et al (2015) Galectin-1 modulates the survival and tumor necrosis factor-related apoptosis-inducing ligand (TRAIL) sensitivity in human hepatocellular carcinoma cells Cancer Biother Radiopharm 30(8) 336-41 DOI: 10.1089/cbr.2015.1857 PMID: 26348206

71. Wei RC et al (2011) Augmenting the antitumor effect of TRAIL by SOCS3 with double-regulated replicating oncolytic adenovirus in hepatocellular carcinoma Hum Gene Ther 22(9) 1109-19 DOI: 10.1089/hum.2010.219 PMID: 21361790

72. Song KY et al (2010) Antitumor efficacy of viral therapy using genetically engineered Newcastle disease virus [NDV(F3aa)GFP] for peritoneally disseminated gastric cancer J Mol Med (Berl) 88(6) 589-96 DOI: 10.1007/s00109-010-0605-6

73. Elankumaran S, Rockemann D and Samal SK (2006) Newcastle disease virus exerts oncolysis by both intrinsic and extrinsic caspase-dependent pathways of cell death J Virol 80(15) 7522-34 DOI: 10.1128/JVI.00241-06 PMID: 16840332 PMCID: 1563725

74. Bai FL et al (2014) Genetically engineered Newcastle disease virus expressing interleukin-2 and TNF-related apoptosis-inducing ligand for cancer therapy Cancer Biol Ther 15(9)1226-38 DOI: 10.4161/cbt.29686 PMID: 24971746 PMCID: 4128865

75. De Miguel D et al (2015) Liposome-bound TRAIL induces superior DR5 clustering and enhanced DISC recruitment in histiocytic lymphoma U937 cells Leuk Res 39(6) 657-66 DOI: 10.1016/j.leukres.2015.03.019 PMID: 25882551

76. Sun X et al (2012) Co-delivery of pEGFP-hTRAIL and paclitaxel to brain glioma mediated by an angiopep-conjugated liposome Biomaterials 33(3) 916-24

77. Dodds $\mathrm{E}$ et al (1999) Cationic lipids and polymers are able to enhance adenoviral infection of cultured mouse myotubes J Neurochem 72(5) 2105-12 DOI: 10.1046/j.1471-4159.1999.0722105.x PMID: 10217291

78. Kasman LM et al (2009) Polymer-enhanced adenoviral transduction of CAR-negative bladder cancer cells Mol Pharm 6(5) 1612-9 DOI: 10.1021/mp9000958 PMID: 19655763 PMCID: $\underline{2757475}$

79. Schwarze SR et al (1999) In vivo protein transduction: delivery of a biologically active protein into the mouse Science

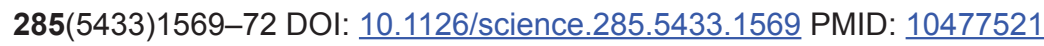

80. Vives E, Brodin P and Lebleu B (1997) A truncated HIV-1 Tat protein basic domain rapidly translocates through the plasma membrane and accumulates in the cell nucleus J Biol Chem 272(25)16010-7 DOI: 10.1074/jbc.272.25.16010 PMID: 9188504

81. Li JF et al (2010) Induction of apoptosis by gene transfer of human TRAIL mediated by arginine-rich intracellular delivery peptides Anticancer Res 30(6) 2193-202 PMID: 20651369

82. Cao L et al (2008) Enhancement of antitumor properties of TRAIL by targeted delivery to the tumor neovasculature Mol Cancer Ther 7(4) 851-61 DOI: 10.1158/1535-7163.MCT-07-0533 PMID: 18413798

83. Wang $X$ et al (2015) Reducible chimeric polypeptide consisting of octa-D-arginine and tetra-L-histidine peptides as an efficient gene delivery vector Int J Nanomedicine 10 4669-90 PMID: 26229469 PMCID: $\underline{4516254}$

84. Kim TH et al (2011) PEGylated TNF-related apoptosis-inducing ligand (TRAIL)-loaded sustained release PLGA microspheres for enhanced stability and antitumor activity J Control Release 150(1) 63-9 DOI: 10.1016/j.jconrel.2010.10.037 
85. Ajorlou E, Khosroushahi AY and Yeganeh H (2016) Novel Water-Borne Polyurethane Nanomicelles for Cancer Chemotherapy: Higher Efficiency of Folate Receptors Than TRAIL Receptors in a Cancerous Balb/C Mouse Model Pharm Res DOI: 10.1007/ s11095-016-1884-6 PMID: 26908046

86. Bagó JR et al (2016) Therapeutically engineered induced neural stem cells are tumour-homing and inhibit progression of glioblastoma Nat Commun 710593 DOI: 10.1038/ncomms10593 PMID: 26830441 PMCID: 4740908

87. Ciavarella $S$ et al (2011) Mesenchymal stem cells: a new promise in anticancer therapy Stem Cells Dev 20(1) 1-10 DOI: $10.1089 /$ $\underline{\text { scd. } 2010.0223}$

88. Akimoto $\mathrm{K}$ et al (2013) Umbilical cord blood-derived mesenchymal stem cells inhibit, but adipose tissue-derived mesenchymal stem cells promote, glioblastoma multiforme proliferation Stem Cells and Development 22(9) 1370-86 DOI: 10.1089/ scd.2012.0486 PMCID: $\underline{3696928}$

89. Sage EK et al (2014) Systemic but not topical TRAIL-expressing mesenchymal stem cells reduce tumour growth in malignant mesothelioma Thorax 69(7) 638-47 DOI: 10.1136/thoraxjnl-2013-204110 PMID: 24567297 PMCID: 4078753

90. Grisendi G et al (2015) Mesenchymal progenitors expressing TRAIL induce apoptosis in sarcomas Stem Cells 33(3) 859-69 DOI: $10.1002 /$ stem.1903

91. Lathrop MJ et al (2015) Antitumor effects of TRAIL-expressing mesenchymal stromal cells in a mouse xenograft model of human mesothelioma Cancer Gene Ther 22(1) 44-54 DOI: 10.1038/cgt.2014.68

92. Nesterenko I et al (2012) Evaluating the effect of therapeutic stem cells on TRAIL resistant and sensitive medulloblastomas PLoS One 7(11) e49219 DOI: $10.1371 /$ journal.pone.0049219 PMID: 23145127 PMCID: $\underline{3492275}$

93. Ciavarella $S$ et al (2012) In vitro anti-myeloma activity of TRAIL-expressing adipose-derived mesenchymal stem cells $\mathrm{Br} J$ Haematol 157(5) 586-98 DOI: 10.1111/j.1365-2141.2012.09082.x PMID: 22420897

94. Loebinger MR et al (2010) TRAIL-expressing mesenchymal stem cells kill the putative cancer stem cell population Br J Cancer 103(11) 1692-7 PMID: 21063402 PMCID: 2994223

95. Kim SM et al (2008) Gene therapy using TRAIL-secreting human umbilical cord blood-derived mesenchymal stem cells against intracranial glioma Cancer Res 68(23) 9614-23 DOI: 10.1158/0008-5472.CAN-08-0451 PMID: 19047138

96. Yu R et al (2013) Delivery of sTRAIL variants by MSCs in combination with cytotoxic drug treatment leads to p53-independent enhanced antitumor effects Cell Death Dis 4 e503 DOI: 10.1038/cddis.2013.19 PMID: 23429289 PMCID: 3734822

97. Yan C et al (2014) Suppression of orthotopically implanted hepatocarcinoma in mice by umbilical cord-derived mesenchymal stem cells with sTRAIL gene expression driven by AFP promoter Biomaterials 35(9) 3035-43 DOI: 10.1016/j.biomaterials.2013.12.037 PMID: $\underline{24406219}$

98. Deng Q et al (2014) TRAIL-secreting mesenchymal stem cells promote apoptosis in heat-shock-treated liver cancer cells and inhibit tumor growth in nude mice Gene Ther 21(3) 317-27 DOI: 10.1038/gt.2013.88 PMID: 24451114

99. Loebinger MR et al (2009) Mesenchymal Stem Cell delivery of TRAIL can eliminate Metastatic Cancer Cancer Res 69(10) 4134-42 DOI: 10.1158/0008-5472.CAN-08-4698 PMID: 19435900 PMCID: 2699841

100. Yulyana $\mathrm{Y}$ et al (2013) Carbenoxolone enhances TRAlL-induced apoptosis through the upregulation of death receptor 5 and inhibition of gap junction intercellular communication in human glioma Stem Cells Dev 22(13) 1870-82 DOI: 10.1089/ scd.2012.0529 PMID: $\underline{23428290}$ PMCID: $\underline{3685394}$

101. Zhang B et al (2012) The inhibitory effect of MSCs expressing TRAIL as a cellular delivery vehicle in combination with cisplatin on hepatocellular carcinoma Cancer Biol Ther 13(12) 1175-84 DOI: 10.4161/cbt.21347 PMID: 22922789 PMCID: 3469475

102. Kim SM et al (2012) Effective combination therapy for malignant glioma with TRAIL-secreting mesenchymal stem cells and lipoxygenase inhibitor MK886 Cancer Res 72(18) 4807-17 DOI: 10.1158/0008-5472.CAN-12-0123 PMID: 22962275 
103. Kim SM et al (2014) Potential application of temozolomide in mesenchymal stem cell-based TRAIL Gene therapy against malignant glioma Stem Cells Transl Med 3(2) 172-82 DOI: 10.5966/sctm.2013-0132 PMID: 24436439 PMCID: 3925057

104.Kaczorowski A et al (2016) Delivery of improved oncolytic adenoviruses by mesenchymal stromal cells for elimination of tumorigenic pancreatic cancer cells Oncotarget 2016 PMID: 26824985 PMCID: 4891025

105. Maeda $\mathrm{H}$ et al (2000) Tumor vascular permeability and the EPR effect in macromolecular therapeutics: a review $J$ Control Release 65(1-2) 271-84 DOI: 10.1016/S0168-3659(99)00248-5 PMID: 10699287

106. Bertrand $\mathrm{N}$ et al (2014) Cancer nanotechnology: the impact of passive and active targeting in the era of modern cancer biology Adv Drug Deliv Rev 66 2-25 DOI: 10.1016/j.addr.2013.11.009 PMCID: 4219254

107. Suri SS, Fenniri H and Singh B (2007) Nanotechnology-based drug delivery systems J Occup Med Toxicol 2 16 DOI: 10.1186/17456673-2-16 PMID: 18053152 PMCID: 2222591

108. Niidome T et al (2006) PEG-modified gold nanorods with a stealth character for in vivo applications $J$ Control Release 114(3) 343-7 DOI: $\underline{10.1016 / j . j c o n r e l .2006 .06 .017}$ PMID: 16876898

109. Wang $\mathrm{K}$ et al (2015) Nanoparticle-mediated target delivery of TRAIL as gene therapy for tlioblastoma Adv Healthc Mater 4(17) 2719-26 DOI: 10.1002/adhm.201500563 PMID: 26498165 PMCID: $\underline{4715716}$

110. Perlstein B et al (2013) TRAIL conjugated to nanoparticles exhibits increased anti-tumor activities in glioma cells and glioma stem cells in vitro and in vivo Neuro Oncol 15(1) 29-40 DOI: $10.1093 /$ neuonc/nos248 PMCID: $\underline{3534416}$

111. Wang $Y$ et al (2015) Triazine-modified dendrimer for efficient TRAIL gene therapy in osteosarcoma Acta Biomater 17 115-24 DOI: 10.1016/j.actbio.2015.01.007 PMID: 25595474

112. Huang RQ et al (2007) Efficient gene delivery targeted to the brain using a transferrin-conjugated polyethyleneglycol-modified polyamidoamine dendrimer Faseb J 21(4) 1117-25 DOI: 10.1096/fj.06-7380com PMID: 17218540

113. Gao S et al (2016) Plasmid pORF-hTRAIL targeting to glioma using transferrin-modified polyamidoamine dendrimer Drug Des Devel Ther 10 1-11 PMID: 26719669 PMCID: $\underline{4690643}$

114. Ediriwickrema A et al (2014) Multi-layered nanoparticles for combination gene and drug delivery to tumors Biomaterials 35(34) 9343-54 DOI: 10.1016/j.biomaterials.2014.07.043 PMID: 25112935 PMCID: 4155034

115. Min SY et al (2015) Facile one-pot formulation of TRAIL-embedded paclitaxel-bound albumin nanoparticles for the treatment of pancreatic cancer Int J Pharm 494(1) 506-15 DOI: 10.1016/j.jijpharm.2015.08.055 PMID: 26315118

116. Thao LQ et al (2016) Doxorubicin-bound albumin nanoparticles containing a TRAIL protein for targeted treatment of colon cancer Pharm Res 33(3) 615-26 DOI: 10.1007/s11095-015-1814-z

117. Guo L et al (2012) Combination of TRAIL and actinomycin D liposomes enhances antitumor effect in non-small cell lung cancer Int J Nanomedicine 7 1449-60 PMID: 22619505 PMCID: $\underline{356168}$

118. Gu G et al (2013) PEG-co-PCL nanoparticles modified with MMP-2/9 activatable low molecular weight protamine for enhanced targeted glioblastoma therapy Biomaterials 34(1) 196-208 DOI: 10.1016/j.biomaterials.2012.09.044

119. Sun NF et al (2012) Nanoliposome-mediated FL/TRAIL double-gene therapy for colon cancer: in vitro and in vivo evaluation Cancer Lett 315(1) 69-77 DOI: 10.1016/j.canlet.2011.10.010

120. Hu Q et al (2016) Tumor microenvironment-mediated construction and deconstruction of extracellular drug-delivery depots Nano Lett 16(2) 1118-26 DOI: $\underline{10.1021 / a c s . n a n o l e t t .5 b 04343}$ PMID: 26785163

121. Reya T and Clevers H (2005) Wnt signalling in stem cells and cancer Nature 434(7035) 843-50 DOI: 10.1038/nature03319 PMID: 15829953

122.Patel A, Cholkar K and Mitra AK (2014) Recent developments in protein and peptide parenteral delivery approaches Ther Deliv 5(3) 337-65 DOI: 10.4155/tde.14.5 PMID: 24592957 PMCID: 4130463 